\title{
Recurrent Nonconvulsive Status Epilepticus in a Patient with Coffin-Lowry Syndrome
}

\author{
Markus Gschwind ${ }^{\mathrm{a}} \quad$ Giovanni Foletti $^{\mathrm{a}, \mathrm{b}} \quad$ Alessandra Baumer ${ }^{\mathrm{c}} \quad$ Armand Bottani $^{\mathrm{d}}$ \\ Jan Novy ${ }^{a}$ \\ aDepartment of Clinical Neuroscience, Centre Hospitalier Universitaire Vaudois, University of Lausanne, Lausanne, \\ ${ }^{b}$ Institution de Lavigny, Lavigny, 'Institute of Medical Genetics, University of Zurich, Schlieren-Zurich, and \\ dService of Genetic Medicine, Geneva University Hospitals, Geneva, Switzerland
}

\section{Key Words}

Coffin-Lowry syndrome - Nonconvulsive status epilepticus . RPS6KA3 mutation c.575G >C (p.R192T) · Stimulus-induced drop episodes

\begin{abstract}
Coffin-Lowry syndrome (CLS) is a rare neurodevelopmental condition caused by heterogeneous mutations in the RPS6KA3 gene on the $\mathrm{X}$ chromosome, leading to severe intellectual disability and dysmorphism in men, while women are carriers and only weakly affected. CLS is well known for stimulus-induced drop episodes; however, epilepsy is not commonly reported in this condition. We report on a CLS patient presenting with recurrent episodes of nonconvulsive status epilepticus (NCSE) with generalized epileptic activity, for which investigations did not find any other cause than the patient's genetic condition. This case underlines that the possibility of nonconvulsive epileptic seizures and status epilepticus should, therefore, be considered in those patients. The treatable diagnosis of NCSE may easily be overlooked, as symptoms can be unspecific.
\end{abstract}

(c) 2015 S. Karger AG, Basel
(C) 2015 S. Karger AG, Base

$1661-8769 / 15 / 0062-0091 \$ 39.50 / 0$
Coffin-Lowry syndrome (CLS) is an X-linked dominant syndrome characterized in males by severe intellectual disability, short stature, facial dysmorphism, peculiar hands (short, soft and fleshy), and progressive kyphoscoliosis [Lowry et al., 1971]. It is caused by highly heterogeneous loss-of-function mutations in the RPS6KA3 gene located on Xp22.12 [Pereira et al., 2010]. Heterozygous females are less severely affected. About $20 \%$ of individuals with CLS present stimulus-induced drop episodes (SIDEs) which typically start from middle childhood to the teens [Stephenson et al., 2005], and 5\% of individuals are also reported to be affected by epileptic seizures [Touraine et al., 2002; Hahn and Hanauer, 2012]. To the best of our knowledge, this is the first description of status epilepticus in CLS. We report on an adult patient presenting with recurrent episodes of nonconvulsive status epilepticus (NCSE).

\section{Clinical Report}

The male patient was first admitted to hospital at the age of 45 years because of a sudden episode of fluctuating decreased alertness; a second, very similar episode recurred one year later. He is an orphan living in an institution, and both his family and

\section{KARGER 125}

E-Mail karger@karger.com www.karger.com/msy
Markus Gschwind, MD

Department of Clinical Neuroscience, Centre Hospitalier Universitaire Vaudois University of Lausanne

Rue du Bugnon 46, CH-1011 Lausanne (Switzerland)

E-Mail markus.gschwind@gmail.com 

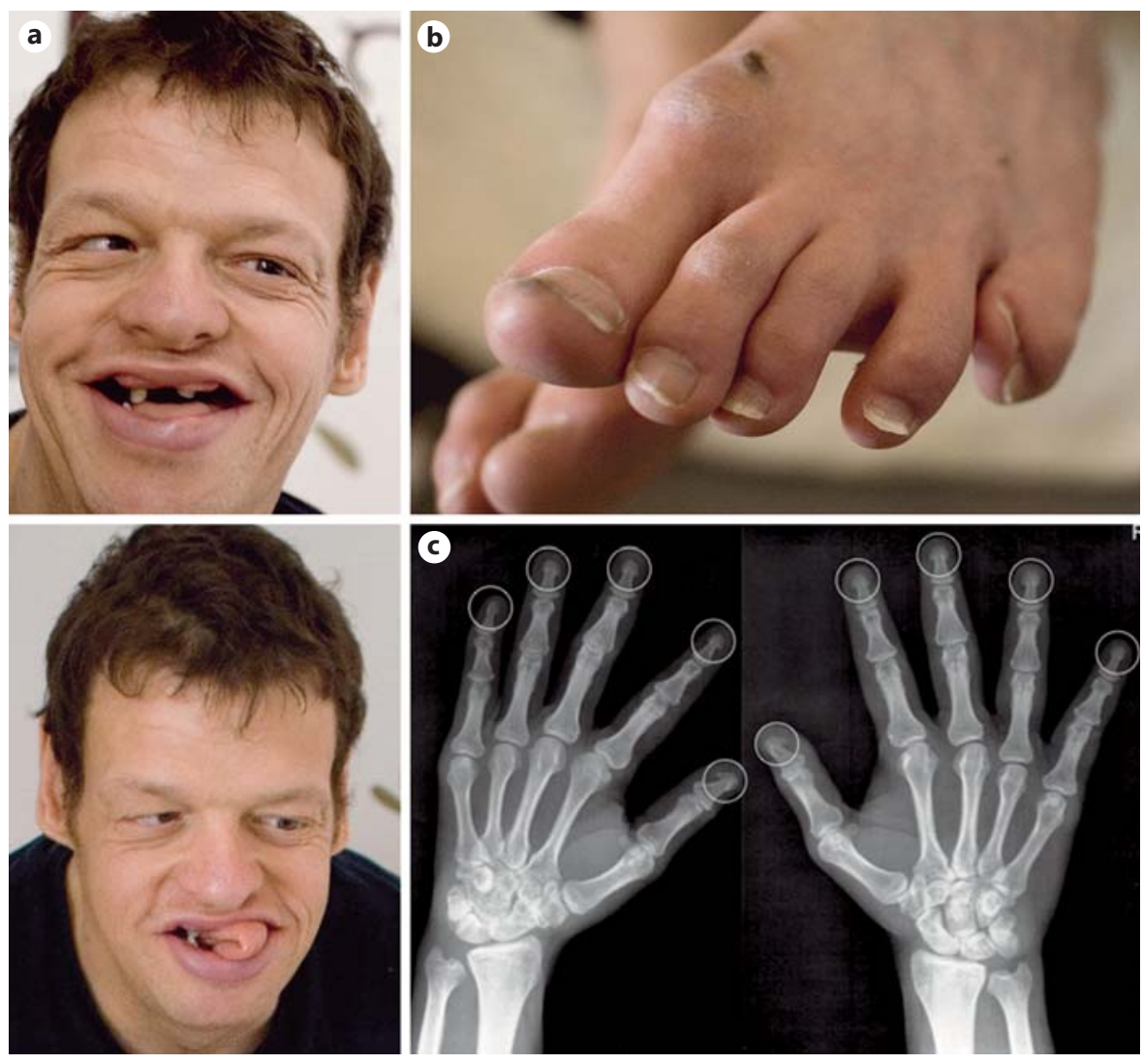

Fig. 1. a The patient at the age of 45 years. Note the facial dysmorphisms with coarse facial traits, slightly downslanting palpebral fissures, a long, folded tongue, and a thick everted lower lip. b Postaxial polydactyly of the patient's left foot. c X-rays of the patient's hands showing the so-called tufting of all distal phalanges (circles).

past medical history are very poorly documented. Allegedly, he suffered from marked learning disabilities since early childhood. The first available medical records at the age of 37 years report severe mental handicap, but no other medical history. Upon examination, the patient presented with a happy and quiet demeanor, was of short stature $(158 \mathrm{~cm})$, had a severe kyphoscoliosis, and had no verbal communication. He had dysmorphic features with coarse facial traits, slightly downslanting palpebral fissures, a short philtrum, a long tongue, hypodontia, and a thick everted lower lip. (fig. 1a). Postaxial polydactyly was noted on his left foot (fig. 1b), and his fingers were neither particularly stubby nor tapering, as often described in textbooks. For several years, the patient had been experiencing attacks of very sudden lower limb weakness, especially when he got surprised, making him fall down. He needed walking assistance because of an ataxic gait. $\mathrm{He}$ never experienced epileptic seizures previously. Both episodes presented with repeated losses of contact of $\sim 30 \mathrm{~s}$, occurring 20 times over $2 \mathrm{~h}$, with tonic head movement to the left, subtle hand myoclonus and purposeless arm movements, profuse sweating and salivation, and associated with urinary incontinence. At admission, he continued to experience episodes of head movements to the left with decreased awareness, as he underwent a videoEEG (fig. 2a). The recording showed generalized continuous ictal activity (discharges of polyspikes) which resolved after administration of $1 \mathrm{mg}$ of intravenous clonazepam (fig. 2b). The patient was then loaded with $1,000 \mathrm{mg}$ of levetiracetam $(20 \mathrm{mg} / \mathrm{kg}$ intra- venous). The work-up did not disclose any other known cause of status epilepticus. Brain MRI showed no major abnormality, but some minor unspecific punctiform T2-hyperintensities in the frontal white matter and moderately enlarged lateral ventricles (most notably on the posterior horns). The patient was discharged on levetiracetam $750 \mathrm{mg}$ per os daily. A year later, while the treatment of levetiracetam was continued at the same dosage, very similar episodes suddenly occurred again with varying intensity. Upon admission to the hospital, he was administered an intravenous loading dose of $1,000 \mathrm{mg}$ levetiracetam, which stopped the seizures. The patient recovered rapidly, and the following day, his EEG was devoid of epileptiform activity. Ictal levetiracetam serum level upon admission was detectable at 32 $\mu \mathrm{mol} / \mathrm{l}$, i.e. below the reference range, but in keeping with the relatively low dose he had been taking. Consequently, the dosage was increased to $1,250 \mathrm{mg}$ daily. When the patient experienced another similar episode of NCSE while on monotherapy, and no triggering factor was identified for this second episode, the treatment was intensified with the addition of another wide-spectrum antiepileptic drug (valproate $500 \mathrm{mg}$ daily). After the second episode, the diagnosis of CLS was postulated upon review of the patient's photographs. X-rays of the hands were performed and showed the typical tufted drumstick appearance of distal phalanges (fig. 1c). Genetic analysis of the RPS6KA3 gene confirmed the diagnosis. 
Fig. 2. EEG of the first episode (average montage). a Ictal recording was marked by subcontinuous bursts of polyspikes and intermingled diffuse spike waves (1-3 s) without clear temporal correlation with the head movements. Background activity was diffusely slow. b After treatment, the recording showed only slow low-voltage background activity. b

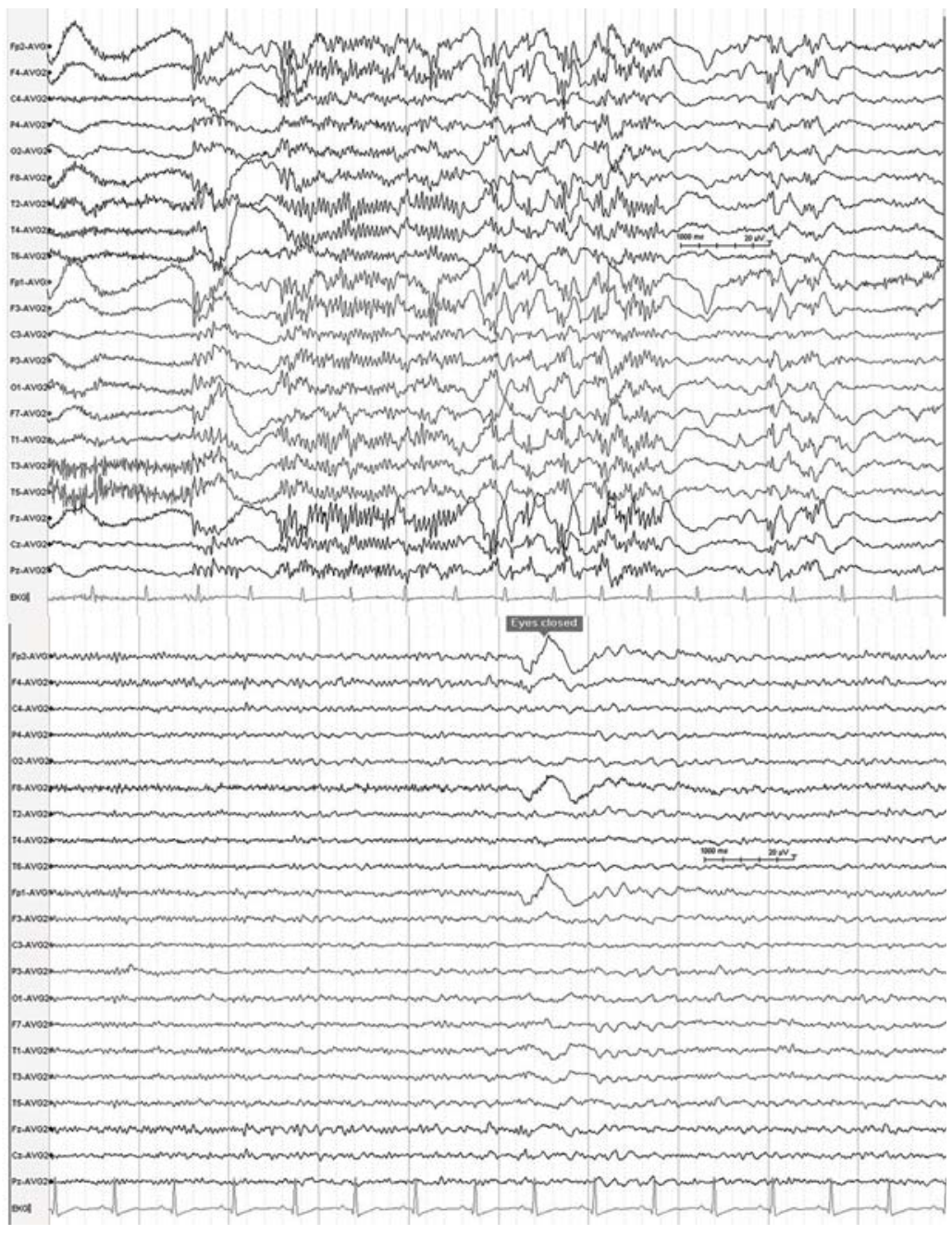

\section{Genetic Analysis}

Molecular analysis of RPS6KA3 (ribosomal protein S6 kinase, $90 \mathrm{kDa}$, polypeptide 3; OMIM 300075) showed a previously undescribed hemizygous mutation c.575G >C (p.R192T). Given the high degree of conservation of the mutated nucleotide and the type of amino acid change, it was considered that this variant was most likely the cause of the patient's phenotype (together with the following prediction scores for clinical significance: Mutation Taster: 'disease causing', probability = 1.0; PolyPhen-2: 'probably damaging', score: 1.000; SIFT: 'tolerated', score 0.19). Unfortunately, his mother had passed away in 1971 , and no maternal relatives were available to study the segregation of this mutation.

NCSE in a Patient with Coffin-Lowry Syndrome

\section{Discussion}

Our patient was diagnosed with CLS very late in his life. Retrospective diagnoses of childhood conditions in adults living in institutions can be challenging at times because early medical records are rarely available and parents may have deceased. His clinical and X-ray features were compatible with previous reports on CLS [O’Riordan et al., 2006; Wang et al., 2006], which prompted molecular genetic diagnosis. CLS is well known for paroxysmal SIDEs. Their pathophysiology is not clear so far. They have been termed cataplexy, hyperekplexia, 
nonepileptic collapses with atonia, or exaggerated startle responses [Havaligi et al., 2007]. Others have described them as cataplexy-like events, characterized by sudden quadriceps hypotonia with subsequent collapse, or hyperekplexia-like episodes, with a startle response to mostly auditory or tactile stimuli [Hahn and Hanauer, 2012]. SIDEs are widely considered as nonepileptic episodes [Nelson and Hahn, 2003; Hahn and Hanauer, 2012].

Epileptic seizures in CLS are rarely reported. In large series, only $5 \%$ of patients with CLS were described to have epileptic seizures [Hunter, 2002; Stephenson et al., 2005], while other authors question if this truly represents an increased prevalence, as the occurrence of epilepsy is commonly reported in more than $5 \%$ of persons with mild learning disabilities and in up to $24 \%$ of persons with severe learning disabilities [Steffenburg et al., 1995; Lhatoo and Sander, 2001; Hahn and Hanauer, 2012], compared to a prevalence of around $1 \%$ in the general population [Picot et al., 2008]. It is, however, commonly thought that in individuals with learning disabilities, the increased prevalence of epilepsy is related to the fact that both epilepsy and learning disabilities are symptoms of a common underlying condition [Lhatoo and Sander, 2001]. In the specific case of CLS, mutations of RPS6KA3 have wide implications [Sassone-Corsi et al., 1999] but without any direct link to known epileptogenic pathways [Ottman et al., 2010; Allen et al., 2013]. An association with epilepsy was however reported in a family with an Xp22.12 microduplication encompassing RPS6KA3 [Matsumoto et al., 2013]. RPS6KA3, expressed in the brain, although less markedly in the cortex (www. genecards.org) [Rappaport et al., 2014], is the major generator of epileptic seizures. One of the pathways, modulated by the gene, is NMDA receptor activation, a func- tion conceivably involved in the occurrence of seizures [Moldrich et al., 2003].

Our patient presented with recurrent unprovoked NCSE with an EEG showing a peculiar generalized pattern (long runs of polyspikes), typically seen in symptomatic generalized epilepsies [Sutter and Kaplan, 2012], the etiology of which is increasingly demonstrated to be genetic [Allen et al., 2013]. Although the short duration of the repeated clinical episodes may have suggested repeated absence seizures, we underline that recurrent motor manifestations suggestive of focal seizures as well as generalized continuous epileptic activity usually associated with tonic seizures make the best description of the patient as NCSE [Sutter and Kaplan, 2012], further highlighting the peculiarity of those episodes. Investigations did not disclose any alternative cause than the patient's underlying genetic condition. The relatively unspecific semiology of these episodes and absence of convulsions may easily have been overlooked and misdiagnosed as behavioral changes, or as a nonspecific deterioration in the general state of health occurring in parallel to medical complications in a patient with severe learning disabilities. This case highlights that prolonged nonconvulsive seizures and status epilepticus can occur in CLS. Therefore, this possibility should be considered in those patients in cases of altered behavior or a decreased general state of health, even though seizures per se are not a frequently reported feature of CLS.

\section{Acknowledgements}

We are grateful to the patient and his caregivers for their cooperation and would like to especially thank Prof. Anita Rauch from the Institute of Medical Genetics in Zurich for making the molecular analysis of RPS6KA3 possible.
References

\footnotetext{
-Allen AS, Berkovic SF, Cossette P, Delanty N, Hunter AG: Coffin-Lowry syndrome: a 20-year Dlugos D, et al: De novo mutations in epileptic encephalopathies. Nature 501:217-221 (2013).

Hahn JS, Hanauer A: Stimulus-induced drop episodes in Coffin-Lowry syndrome. Eur J Med Genet 55:335-337 (2012).

Havaligi N, Matadeen-Ali C, Khurana DS, Marks $\mathrm{H}$, Kothare SV: Treatment of drop attacks in Coffin-Lowry syndrome with the use of sodium oxybate. Pediatr Neurol 37:373-374
} (2007). 
Matsumoto A, Kuwajima M, Miyake K, Kojima K, Nakashima N, et al: An Xp22.12 microduplication including RPS6KA3 identified in a family with variably affected intellectual and behavioral disabilities. J Hum Genet 58:755757 (2013).

Moldrich RX, Chapman AG, De Sarro G, Meldrum BS: Glutamate metabotropic receptors as targets for drug therapy in epilepsy. Eur J Pharmacol 476:3-16 (2003).

Nelson GB, Hahn JS: Stimulus-induced drop episodes in Coffin-Lowry syndrome. Pediatrics 111:e197-e202 (2003).

O'Riordan S, Patton M, Schon F: Treatment of drop episodes in Coffin-Lowry syndrome. J Neurol 253:109-110 (2006).

-Ottman R, Hirose S, Jain S, Lerche H, LopesCendes I, et al: Genetic testing in the epilepsies-report of the ILAE Genetics Commission. Epilepsia 51:655-670 (2010).
Pereira PM, Schneider A, Pannetier S, Heron D, Hanauer A: Coffin-Lowry syndrome. Eur J Hum Genet 18:627-633 (2010).

Picot MC, Baldy-Moulinier M, Daurès JP, Dujols P, Crespel A: The prevalence of epilepsy and pharmacoresistant epilepsy in adults: a population-based study in a Western European country. Epilepsia 49:1230-1238 (2008).

Rappaport N, Twik M, Nativ N, Stelzer G, Bahir I, et al: MalaCards: A Comprehensive Automatically-Mined Database of Human Diseases. Curr Protoc Bioinformatics 47:1.24.11.24.19 (2014).

Sassone-Corsi P, Mizzen CA, Cheung P, Crosio C, Monaco L, et al: Requirement of Rsk-2 for epidermal growth factor-activated phosphorylation of histone H3. Science 285:886-891 (1999).

Steffenburg U, Hagberg G, Kyllerman M: Active epilepsy in mentally retarded children. II. Etiology and reduced pre- and perinatal optimality. Acta Paediatr 84:1153-1159 (1995).
Stephenson JB, Hoffman MC, Russell AJ, Falconer J, Beach RC, et al: The movement disorders of Coffin-Lowry syndrome. Brain Dev 27: 108-113 (2005).

Sutter R, Kaplan PW: Electroencephalographic criteria for nonconvulsive status epilepticus: synopsis and comprehensive survey. Epilepsia 53(suppl 3):1-51 (2012).

Touraine RL, Zeniou M, Hanauer A: A syndromic form of X-linked mental retardation: the Coffin-Lowry syndrome. Eur J Pediatr 161: 179-187 (2002).

Wang Y, Martinez JE, Wilson GL, He XY, TuckMuller CM, et al: A novel RSK2 (RPS6KA3) gene mutation associated with abnormal brain MRI findings in a family with CoffinLowry syndrome. Am J Med Genet A 140: 1274-1279 (2006). 